\title{
EVALUATION OF THE MAIN CEOS PSEUDO CALIBRATION SITES USING MODIS BRDF/ALBEDO PRODUCTS
}

\author{
Said Kharbouche ${ }^{\mathrm{a},{ }^{*}, \text { Jan-Peter Muller }}{ }^{\mathrm{a}}$ \\ ${ }^{a}$ Imaging Group, Mullard Space Science Laboratory, University College London, Holmbury St Mary, Surrey, RH56NT, UK \\ (s.kharbouche, j.muller)@ucl.ac.uk
}

Commission I, WG I/3

KEY WORDS: Satellites, Calibration, Multi-Spectral, BRDF/Albedo, MODIS

\begin{abstract}
:
This work describes our findings about an evaluation of the stability and the consistency of twenty primary PICSs (Pseudo-Invariant Calibration Sites). We present an analysis of 13 years of 8-daily MODIS products of BRDF parameters and white-sky-albedos (WSA) over the shortwave band. This time series of WSA and BRDFs shows the variation of the "stability" varies significantly from site to site. Using a 10x10 km window size over all the sites, the change in of WSA stability is around 4\% but the isotropicity, which is an important element in inter-satellite calibration, can vary from $75 \%$ to $98 \%$. Moreover, some PICS, especially, Libya- 4 which is one of the PICS which is most employed, has significant and relatively fast changes in wintertime. PICS observations of BRDF/albedo shows that the Libya-4 PICS has the best performance but it is not too far from some sites such as Libya-1 and Mali. This study also reveals that Niger-3 PICS has the longest continuous period of high stability per year, and Sudan has the most isotropic surface. These observations have important implications for the use of these sites.
\end{abstract}

\section{INTRODUCTION}

Pseudo-Invariant Calibration Sites (PICS) are being employed by the space agencies to calibrate and validate satellite-derived products, starting with Top of Atmosphere (ToA) radiance. A continuous updating of calibration coefficients and uncertainties is essential to detect Earth Observation sensor anomalies and evaluate the degradation of the instruments in order to produce Climate Data Records. As a consequence, bridging between satellites measured radiance and ground level measurements of radiance needs to be properly characterised. Generally, the selection of PICS is based on many criteria such as temporal stability, flat terrain, spatial uniformity, no vegetation cover, intermittent snow, aerosol level, clear sky probability, and the uniformity of reflectance over all view angles (isotropic character). In addition other factors can be considered such as accessibility to carry out ground measurements, and the availability of long-term historical records (Chander et al., 2013).

The main PICS locations were identified in the 1990s using the geostationary weather satellite Meteosat-4 (MVIRI) data over the European/African disk area (Cosnefroy et al., 1996 and 1997). That identification was mainly based on two criteria: 1) their temporal and spatial stability (below 3\%) for top-ofatmosphere reflectance (ToA) during a few months of observation; and 2) the geomorphology of sites such that they are very dry and sand dunes sites were preferred. Twenty PICS locations were identified, all in desert area: three PICSs in the Saudi-Arabia's desert and the others dispersed across the desert of northern Africa. In 2008, the Committee on Earth Observation Satellites (CEOS) Working Group on Calibration/Validation (CEOS-WGCV) recommended that the space agencies should focus on six PICS: Libya-1, Libya-4, Algeria-3, Algeria5, Mauritania1 and Mauritania2. CEOSWGCV justifies this choice by the superiority of these selected sites in terms of temporal and spatial resolutions. Many efforts have been made to assess the suitability of the PICS locations. But most of these are based on level-1 products (radiance or top of atmosphere reflectance). However, these inter-comparisons do not employ atmospherically corrected reflectance and Wu et al, (Wu, 2013), have shown that this choice could contaminate the results. Some works are based on the deployment of highresolution sensors (i.e. Landsat) such as (Helder, 2010) but the range of view zenith angle for these satellites is very narrow and close to nadir. Hence anisotropic effects resulting from BRDF variations are not considered. It is therefore crucial that such anisotropic effects can be studied and the departure from isotropy characterised.

Thus, we propose here the evaluation of PICS by high-level products, namely, White-Sky-Albedo (WSA) albedo and BiDirectional Reflectance Directional Function (BRDF). They are derived from atmospherically corrected surface bi-directional reflectance factors (BRFs) and the BRDF combines many samples of different view and illumination geometries to summarise these anisotropic effects. Both WSA and BRDF is here used in an evaluation of stability, and BRDF will be used in the evaluation of the (an)isotropic character of the surfaces.

The next section presents the methodology that was followed to produce the statistical analysis, and then the results are shown in the following section, before we discuss the results and draw conclusions in the last section.

\section{DATA SELECTION \& STATISTICS}

Twenty (20) PICSs that were identified by Cosnefory et al, (Cosnefory, 1996) and (Cosnefory, 1997), were assessed in this study. Initially, the area of each site varied from $100 \mathrm{~km}^{2}$ to $1000 \mathrm{~km}^{2}$ but we fix here the same size for all PICs in order to eliminate any bias that can be caused by the difference in sizes. The study area for each site is a $10 \times 10 \mathrm{~km}$ area, centred on the site's centre co-ordinates. Table 1 lists the names and geographic coordinates of the 20 PICs; with sites marked by $(*)$ denoting those, which have been selected by CEOS as the most stable sites. 
Two MODIS products of collection 5 have been used in this study: 1) MCD43A1 ${ }^{1}$, which represents the three BRDF parameters $\left(k_{\text {iso }}, k_{\text {geo }}, k_{\text {vol }}\right)$; and 2$) \mathrm{MCD} 43 \mathrm{~A} 3^{2}$, which represents white-sky-albedo (WSA). Another product $\mathrm{MCD} 43 \mathrm{~A} 2^{3}$ that contains quality flags and masks is also used. For more details about these products we refer the reader to (Wanner, 1997); (Strahler, 1999) and (Schaaf, 2002).

The spatial resolution of these products is $500 \times 500 \mathrm{~m}$, and the temporal resolution is 8-daily with a synthesis period of 16-days (observation window to estimate BRDF). The broadband shortwave (SW), is derived from a linear combination of the seven narrow bands of the MODIS land BRDF product. As a result, stability over the SW implies stability over all narrow bands. The family of product parameters contained within MCD43 are derived from data provided by the MODIS instrument on-board the two satellites, Terra (since July 2000) and Aqua (since March 2002). Thus, although the products cited above (MCD43A) are available since March 2002, our time of interest starts on $1^{\text {st }}$ of January 2003 and ends on $31^{\text {st }}$ of December 2015. Consequently, the observation period for this study is 13 complete years. The quality of BRDF and WSA depends on the number of high quality samples from Aqua and Terra, thus that time period allowed us to analyse the data of similar quality, i.e. whenever there was availability of both Terra and Aqua.

\begin{tabular}{|c|c|}
\hline PICS Name & lat,lon \\
\hline Algeria-1 & $23.80^{\circ},-00.40^{\circ}$ \\
\hline Algeria-2 & $26.09^{\circ},-01.38^{\circ}$ \\
\hline Algeria-3* & $30.32^{\circ}, 07.66^{\circ}$ \\
\hline Algeria-4 & $30.04^{\circ}, 05.59^{\circ}$ \\
\hline Algeria-5* & $31.02^{\circ}, 02.23^{\circ}$ \\
\hline Arabia-1 & $18.88^{\circ}, 46.76^{\circ}$ \\
\hline Arabia-2 & $20.13{ }^{\circ} 50.96^{\circ}$ \\
\hline Egypt-1 & $27.12^{\circ}, 26.10^{\circ}$ \\
\hline Egypt-2 & $22.94^{\circ}, 28.79^{\circ}$ \\
\hline Libya-1 & $24.42^{\circ}, 13.35^{\circ}$ \\
\hline Libya-2 & $25.05^{\circ}, 20.48^{\circ}$ \\
\hline Libya-3 & $23.15^{\circ}, 23.10^{\circ}$ \\
\hline Libya-4* & $28.55^{\circ}, 23.39^{\circ}$ \\
\hline Mali & $19.12^{\circ},-04.85^{\circ}$ \\
\hline Mauritania- ${ }^{*}$ & $19.40^{\circ},-09.30^{\circ}$ \\
\hline Mauritania-2 & $20.85^{\circ},-08.78^{\circ}$ \\
\hline Niger-1 & $19.67^{\circ}, 09.81^{\circ}$ \\
\hline Niger-2 & $21.37,^{\circ} 10.59^{\circ}$ \\
\hline Niger-3 & $21.57^{\circ}, 07.96^{\circ}$ \\
\hline Sudan & $21.74{ }^{\circ} 28.22^{\circ}$ \\
\hline
\end{tabular}

Table 1. List of 20 PICSs with geographic coordinates of their centres. The 6 recommended PICS by CEOS are marked by *.

A widow of $10 \times 10 \mathrm{~km}$ pixels is centred to subset the data.

Figure 1 shows WSA (average over 10x10 km) by site every 8days between 2003 and 2015 (13 years). Similarly, Figure 2 shows the coefficient of variation (standard deviation divided by mean) of WSA within a $10 \times 10 \mathrm{~km}$ area (400 resolution cells of $500 \times 500 \mathrm{~m})$, i.e., it represents the spatial homogeneity within a window of observation.

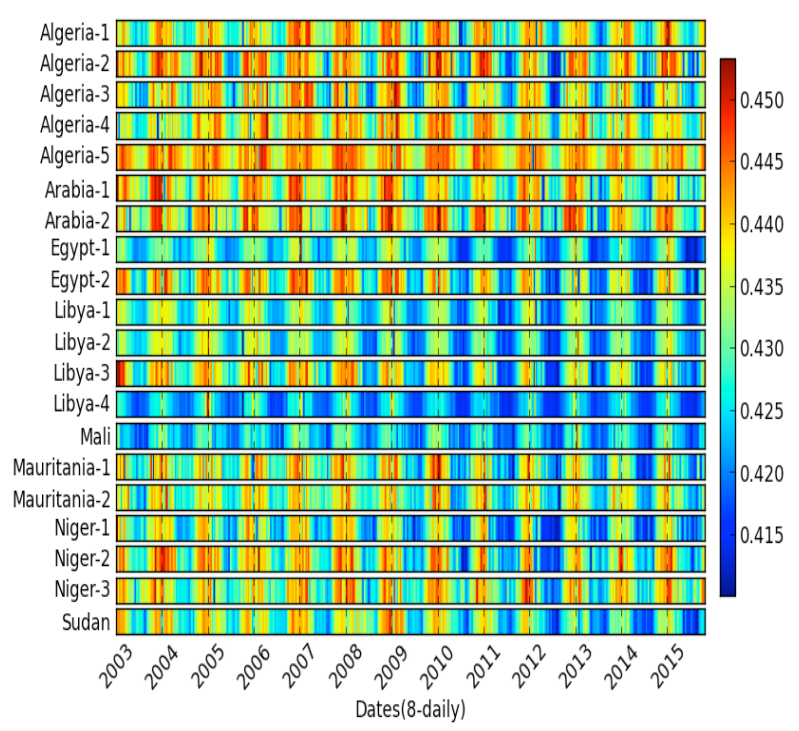

Figure 1. Shortwave White-sky-albedo (WSA) by site, 8 daily for 13 years (2003-2015).

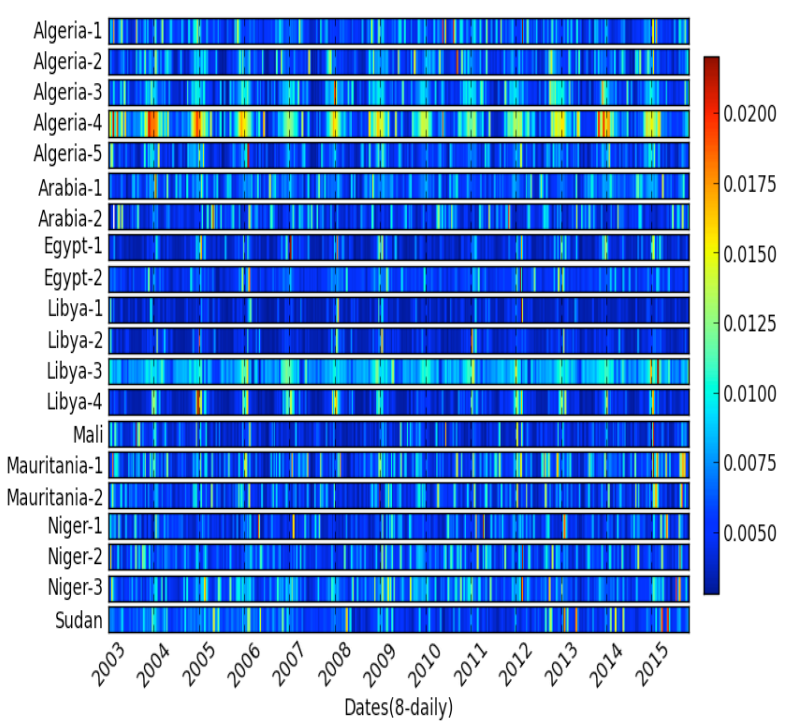

Figure 2. Coefficient of variation of WSA within the $10 \times 10 \mathrm{~km}$ window. It represents the temporal variation of the spatial

homogeneity, 8 daily for 13 years (2003-2015).

The inter-satellite or satellite-PICS calibration over a highly isotropic site can significantly minimize the impact of differences in view angles. So, the isotropicity can be considered one of the most essential criteria in the selection and categorizing PICSs. It indicates how much the reflectance is independent from view and illumination angles. We characterise the degree of isotropicity of a surface by the following formula:

$$
i d x_{i s o}=k_{i s o} /\left(k_{i s o}+k_{\text {geo }}+k_{\text {vol }}\right)
$$

Where $k_{i s o}, k_{g e o}, k_{v o l}$ are three positive values that are given by the product MCD43A1 by pixel, time and band, in such a way the reflectance can be computed as follows:

$$
\rho\left(\theta_{s}, \theta_{v}, \phi\right)=k_{i s o}+k_{v o l} F_{v o l}\left(\theta_{s}, \theta_{v}, \phi\right)+k_{\text {geo }} F_{\text {geo }}\left(\theta_{s}, \theta_{v}, \phi\right)
$$

\footnotetext{
1https://lpdaac.usgs.gov/dataset_discovery/modis/modis_products_table/mcd43a1 ${ }^{2} \mathrm{https} / / / \mathrm{lpdaac} . u s g s . g o v /$ dataset_discovery/modis/modis_products_table/mcd $43 \mathrm{a} 3$
}

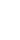


where $\theta_{s}, \theta_{v}$, and $\phi$ represent Solar zenith angle, view zenith angle and relative azimuth angle, respectively; $F_{v o l}($.$) is the$ volume scattering kernel, based on the Ross-Thick approximation; and $F_{\text {geo }}($.$) is the geometric kernel, based on the$ Li-sparse reciprocal function (Strahler et al., 1999).

Thus, we can assume that $i d x_{i s o}$ varies between 0 (totally anisotropic) and 1 (totally isotropic). A surface therefore with a higher $i d x_{i s o}$ can be considered more isotropic.

The Figure 3 shows the time variation of the isotropicity degree $\left(i d x_{i s o}\right)$ for all sites, 8-daily over 13 years (2003-2015).

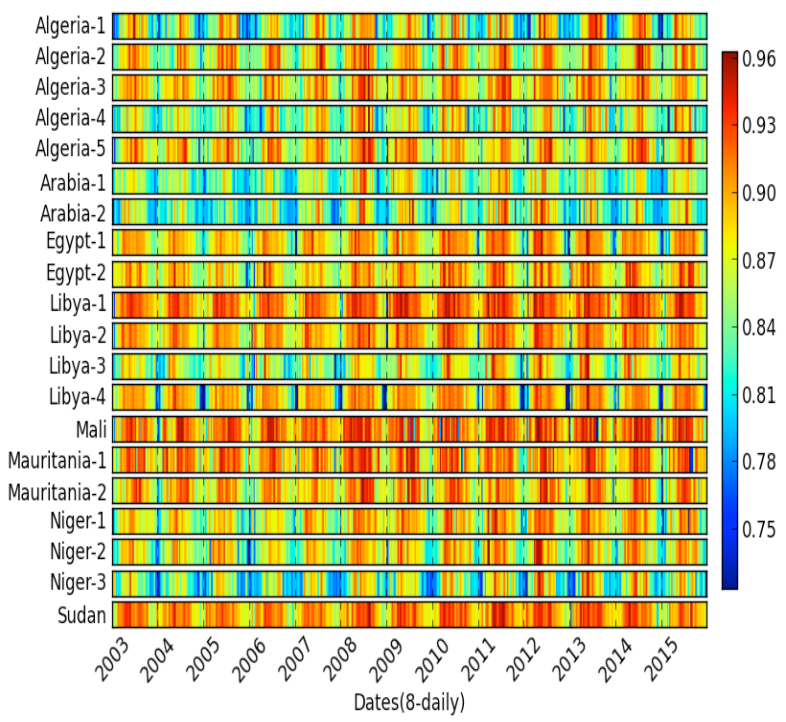

Figure 3. Isotropicity index $\left(k_{i s o} /\left(k_{i s o}+k_{\text {geo }}+k_{\text {vol }}\right)\right)$, with 0 means totally anisotropic and " 1 " means totally isotropic. 8-daily over 13 years $(2003-2015)$.

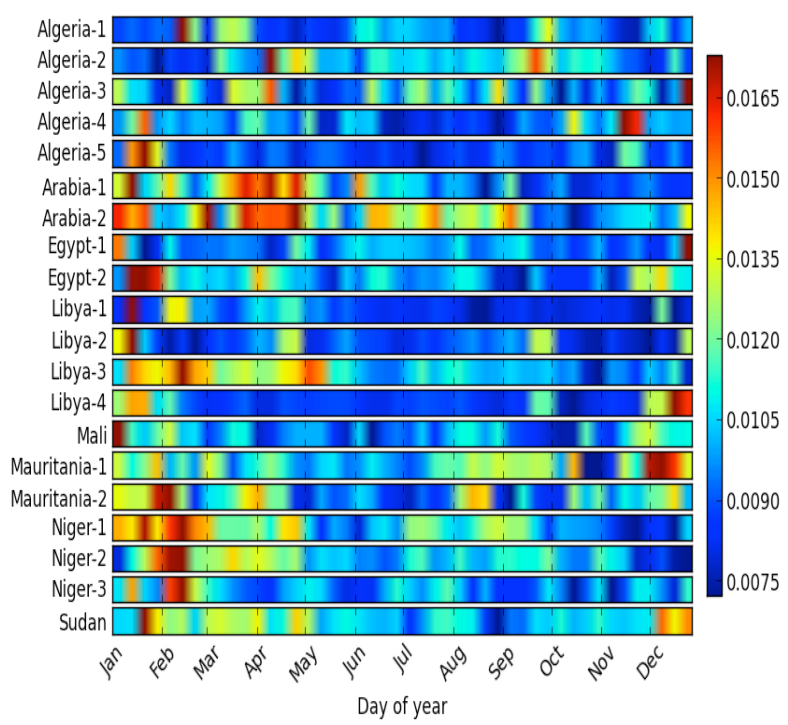

Figure 4. Coefficient of variation of WSA by day of year. Extracted from 13 years of MODIS-WSA: 2003-2015.

Figures 3 and 4 show that there are apparent systematic changes by period of year. So, we compute the coefficient of variation of
WSA for each 8-day of year $(001,009,015 \ldots 361)$ over the same 8-day in all 13 years (Figure 4). In the same manner, we compute the coefficient of variation of isotropicity index $\left(i d x_{i s o}\right)$; see Figure 5.

Finally, the histogram of Figure 6 gives the relative (to the maximum) coefficient of variation of WSA and relative (also to the maximum) isotropicity index.

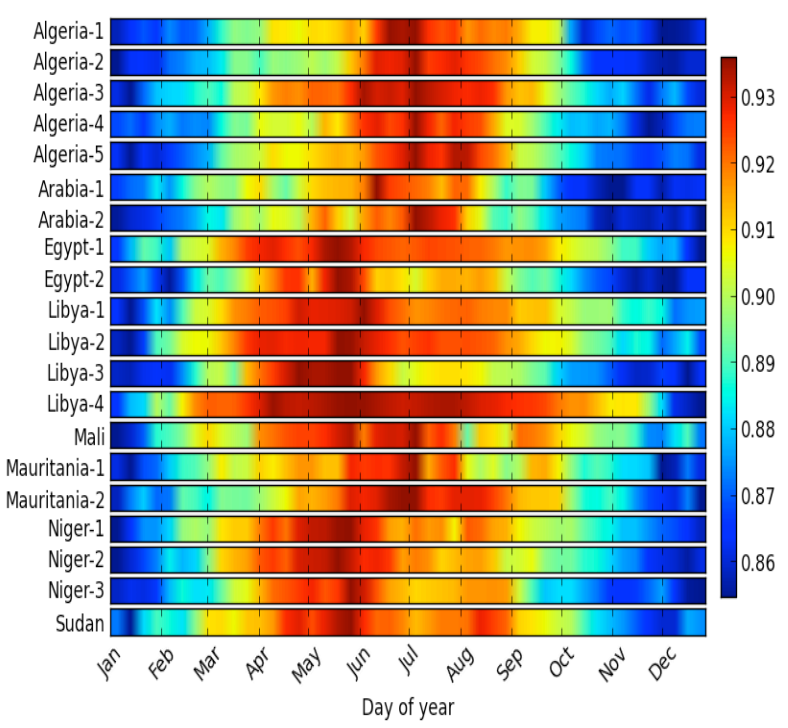

Figure 5. Isotropicity index $\left(k_{i s o} d\left(k_{i s o}+k_{\text {geo }}+k_{\text {vol }}\right)\right)$ by day of year (0: totally anisotropic, 1: totally isotropic). Extracted from 13 years (2003-2015) of 8-daily MODIS-BRDF.

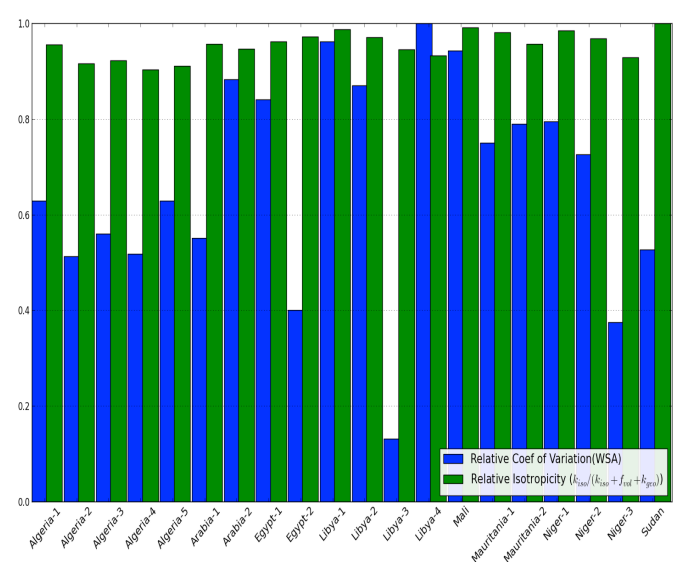

Figure 6. Relative (to the maximum) isotropicity index and relative coefficient of variation (relative to the maximum).

\section{DISCUSSION}

Figure 1 indicates that the WSA appears to have decreased slightly over the last few years $(2-3 \%)$ for some PICSs, especially Sudan, Libya-3 and Egypt-2. But the average variation over 13 years is about $5 \%$; with good stability from Libya-4, Mali and Algeria-5.

The spatial stability of 500x500m pixels within a $10 \times 10 \mathrm{~km}$ area is about $1 \%$ for 13 years with relatively slight seasonal changes. It appears that Libya-1 and Libya-2 have the highest 
spatial stability; whereas on the other hand, Algeria-4 and Libya have the lowest spatial stability (relative).

The isotropicity index varies generally between 0.7 and 0.97 (Figure 3). It is highest for Sudan, Mali and Libya-1. Note that it goes down significantly and seasonally for Libya-4.

The change in the coefficient of variation of WSA over the year (Figure 4) indicates that all sites are stable in summer but Algeria-2 and Algeria-3 have the highest stability during wintertime. However, we can say that Algeria-5 and Egypt-1 have the longest period of stability for WSA.

From Figure 5 we can conclude that the istoropicity is higher in summertime for all sites (95\%); Mali and, notably Sudan have the highest isotropicity per year. Figure 5 shows also that the isotropicity falls a lot for Libya-4 during wintertime (supported also by the results shown in Figure 3)

The histograms shown in Figure 6 indicate that Libya- 4 has the highest stability over the 13 years from observations of WSA. However Sudan, has the most isotropic surface. The 6 selected PICSs by CEOS (Mauritania-1, Mauritania-2, Algeria-1, Algeria-2, Libya-1, Libya-4) contain the tow most stable sites (Libya-4 and Libya-1). However, the stability of Mauritania$1 \& 2$ and Algeria-1\&2 can be compared to some unselected PICSs.

\section{CONCLUSIONS}

In this study, we have evaluated the performances of PICS using time series measurements of BRDF and WSA from MODIS collection-5, which are extracted from atmospherically corrected surface reflectance. We analysed 13 years of data over 20 PICSs with a $10 \times 10 \mathrm{~km}$ observation window, a spatial resolution of $500 \times 500 \mathrm{~m}$ and a temporal resolution of 8-daily. We also evaluated the stability by day of year by analysing the same day over 13 years. The findings here confirm that the stability of PICSs change periodically and seasonally. Commonly, the stabilities reach their maximums during summertime. However, for a few PICSc they also indicate high stability during wintertime. This study also revealed that WSA of some PICSs declined slightly over the last 5-7 years. Note that the six selected PICs by CEOS, largely, have good performance, and include the two most stable PICSs (Libya-1, Libya-4) over whole 13 years and a stable PICS during wintertime (Algeria-3). On the other hand, our analysis suggests the performance changes seasonally, and differently from site to site, which should be taken into consideration in the process of satellite calibration.

This study was based on shortwave data but in future we plan to deploy the seven narrow bands of MODIS in order to evaluate PICS by wavelength. We will also use data from MISR to reveal further effects caused by sensor degradation.

\section{ACKNOWLEDGEMENTS}

We acknowledge support from the EU-FP7 programme under QA4ECV grant agreement $n^{\circ} 607405$.

\section{REFERENCES}

Chander, G., Hewison, T.J., Fox, N., Wu, X., Xiong, X. and Blackwell, W.J., 2013. Overview of intercalibration of satellite instruments. Geoscience and Remote Sensing, IEEE
Transactions on, 51(3), pp.1056-1080.

Cosnefroy, H., Leroy, M. and Briottet, X., 1996. Selection and characterization of Saharan and Arabian desert sites for the calibration of optical satellite sensors. Remote Sensing of Environment, 58(1), pp.101-114.

Cosnefroy, H., Briottet, X., Leroy, M., Lecomte, P. and Santer, R., 1997. A field experiment in Saharan Algeria for the calibration of optical satellite sensors. International Journal of Remote Sensing, 18(16), pp.3337-3359.

Helder, D.L., Basnet, B. and Morstad, D.L., 2010. Optimized identification of worldwide radiometric pseudo-invariant calibration sites. Canadian Journal of Remote Sensing, 36(5), pp.527-539.

Schaaf, C., F. Gao, A. Strahler, W. Lucht, X. Li, T. Tsang, N. Strugnell, X. Zhang, Y. Jin, J. Muller, P. Lewis, M. Barnsley, P. Hobson, M. Disney, G. Roberts, M. Dunderdale, C. Doll, R. d'Entremont, B. Hu, S. Liang, J. Privette, and D. Roy, 2002 First operational BRDF, albedo nadir reflectance products from MODIS. Remote Sensing of Environment, vol. 83, no. 1, pp. 135-148

Strahler, A.H., Muller, J.P., Lucht, W., Schaaf, C.B., Tsang, T., Gao, F., Li, X., Lewis, P. and Barnsley, M.J., 1999. MODIS BRDF/albedo product: algorithm theoretical basis document version 5.0. MODIS documentation, pp.5-37.

Wu, A., Xiong, X., Doelling, D.R., Morstad, D., Angal, A. and Bhatt, R., 2013. Characterization of Terra and Aqua MODIS VIS, NIR, and SWIR spectral bands' calibration stability. IEEE Transactions on,Geoscience and Remote Sensing, 51(7), pp.4330-4338.

Wanner, W., A. Strahler, B. Hu, P. Lewis, J. Muller, X. Li, C. Schaaf, and M. Barnsley, 1997 Global retrieval of bidirectional reflectance and albedo over land from EOS MODIS and MISR data: Theory and algorithm, J Geophys Res-Atmos, vol. 102, no. 14, pp. 17143-17161. 